\title{
Emotion-Regulation in Psychosis: Patients with Psychotic Disorders Apply Reappraisal Successfully
}

\author{
Sandra M. Opoka ${ }^{1}$ (D) Johanna Sundag ${ }^{1} \cdot$ Marcel Riehle $^{1} \cdot$ Tania M. Lincoln $^{1}$
}

Accepted: 30 September 2020 / Published online: 17 October 2020

(c) The Author(s) 2020

\begin{abstract}
Background Emotion-regulation is assumed to be impaired in psychosis: patients with psychotic disorders (PD) self-report to use reappraisal less frequently than healthy controls (HC), but it is uncertain whether they are also less successful in applying reappraisal. Moreover, it has not been investigated whether the habitual use of reappraisal is associated with the ability to use reappraisal successfully.

Methods To address these questions, PD ( $n=60)$, a clinical control group of patients with anxiety disorders (AD; $n=40)$ and $\mathrm{HC}(n=40)$ completed questionnaires on habitual emotion-regulation and alexithymia, performed tests on neurocognitive functioning and jumping-to-conclusion bias, and were subjected to an emotion-regulation paradigm, in which they were asked to down-regulate induced anxiety and sadness.

Results No differences were found between PD and HC regarding the habitual use of reappraisal, but PD reported to use reappraisal more frequently than $\mathrm{AD}$. All groups were able to down-regulate anxiety and sadness via reappraisal following instructions. The habitual use of reappraisal and the reduction of anxiety and sadness after instruction were uncorrelated.

Conclusion These findings question the common assumption that PD use reappraisal less often or less successfully. At least under laboratory condition, PD do not appear to be impaired in reducing negative affect via reappraisal successfully.
\end{abstract}

Keywords Psychosis $\cdot$ Schizophrenia $\cdot$ Emotion regulation $\cdot$ Reappraisal

High rates of depression and anxiety are found in patients with psychotic disorders (PD; meta-analyses: Achim et al. 2011; Li et al. 2020). Negative affect, such as depression and anxiety, is associated with lower quality of life (e.g. Gardsjord et al. 2016; Huppert et al. 2001) and is postulated to contribute to the formation and maintenance of psychotic symptoms (Garety et al. 2001; Hartley et al. 2013). Thus, it does not seem surprising that psychosis is also associated with deficits in emotion-regulation (Ludwig et al. 2019), which was originally defined as "extrinsic and intrinsic processes responsible for monitoring, evaluating, and modifying emotional reactions, especially their intensive and temporal features, to accomplish one's goals" (Thompson 1994, pp.27-28) and has undergone numerous revisions

Sandra M. Opoka

sandra.martha.opoka@uni-hamburg.de

1 Section for Clinical Psychology and Psychotherapy, Institute of Psychology, Faculty of Psychology and Movement Sciences, Universität Hamburg, Von-Melle-Park 5, 20146 Hamburg, Germany and extensions since then (e.g. Gross 2015; Hofmann 2014; Hofmann et al. 2012; Koole 2009; Tamir et al. 2015). In this study we focused on one specific emotion-regulation strategy, namely reappraisal.

Reappraisal in the context of emotion regulation is commonly defined as cognitively transforming a situation in order to change its emotional impact (Gross 1998). For example, being passed by a colleague on the street without being greeted could spontaneously be interpreted as being disliked by that person, which may cause a person to feel sad or angry on a subjective level and may also be associated with higher levels of arousal, which may then be evaluated as threatening (Freeman et al. 2002). However, being able to then consider alternative explanations, such as the colleague being in a hurry, could buffer the initial emotional reaction. Reappraisal has been found to be effective on a short-term basis in the reduction of negative affect in experimental designs and the habitual use of reappraisal has been found to be functional in terms of being associated with adaptive long-term outcomes, such as healthier patterns of affect, social functioning, and well-being in healthy adults 
(John \& Gross 2004; McRae et al. 2012a). An effect of reappraisal has also been demonstrated on physiological indicators of arousal (e.g. Hofmann et al. 2009; Jackson et al. 2000; McRae et al. 2012b; Ray et al. 2010).

Over and beyond the observation that negative affect is high in people with psychosis, there are several reasons to expect PD to have pronounced difficulties in using reappraisal successfully: First, the ability to identify and describe one's emotions, which is considered a prerequisite of effective reappraisal (Barrett et al. 2001; Füstös et al. 2012; Van Rijn et al. 2011), has been found to be impaired in psychosis (alexithymia, O'Driscoll et al. 2014). Second, deficits in neurocognitive functioning could hamper the successful implementation of a cognitively demanding strategy such as reappraisal (Schmeichel and Tang 2015). Third, acute psychosis is associated with a tendency to jump to conclusions, i.e. making unwarranted decisions on the basis of little evidence (Dudley et al. 2015). This cognitive bias is also likely to antagonize attempts to generate and decide on helpful alternative explanations and therefore likely to impede reappraisal. Nevertheless, it remains to be shown whether these factors are indeed related to the ability to apply reappraisal successfully.

Previous research on emotion-regulation and psychosis has mostly focused on the self-reported habitual use of reappraisal. In these studies, participants are typically asked to report if or how often they use reappraisal. A meta-analysis of questionnaire-based studies found that PD report to use reappraisal habitually to a lesser extent compared to healthy control subjects (HC) with a small to moderate effect size (Ludwig et al. 2019), but studies comparing PD to clinical control groups are scarce. This is surprising, given that emotion-regulation deficits are prevalent in numerous other disorders, particularly in affective and anxiety disorders (PicóPérez et al. 2017). Thus, comparing PD to patients with these disorders is of interest in order to assess psychosisspecificity. Also, a problem with the types of questionnaires used is that they tend not to assess success but are restricted to the analysis of how frequently certain emotion-regulation strategies are used due to the way the questions are phrased and scaled (e.g. item from the Cognitive Emotion Regulation Questionnaire (CERQ): 'I look for the positive sides to the matter' with a scale ranging from 'never' to 'always' Garnefski et al. 2002). However, the amount of using reappraisal does not necessarily reveal information about the ability to successfully implement the strategy. To assess this ability, we need to measure the reduction in negative affect following the use of a strategy.

To date, only few studies have used experimental designs to examine the successful application of reappraisal in PD. These studies typically use emotion-eliciting stimuli, such as pictures, to induce emotions and subjects are asked to either apply certain emotion-regulation strategies (experimental conditions) or to just view (control condition) and to rate their subjective emotion intensity. Three such studies found no deficits in the ability to implement reappraisal successfully in PD (Grezellschak et al. 2015; Painter et al. 2019; Perry et al. 2012). Only one study found evidence for potential deficits in reappraisal success, but this study compared subclinical participants with high delusion-proneness to participants with low delusions-proneness (Westermann et al. 2014). Beyond the low number of available studies, a problem with those at hand is that they predominantly (1) comprise small samples that are not suited to detect small effects, (2) do not include more than one indicator of arousal, which is important as emotion regulation is assumed to have an impact on all levels of emotional responding (Koole, 2009) (3) do not include more than one emotion to examine emotion-specificity, even though a predictive value of affect on psychotic symptoms has predominantly been found for anxiety (Freeman et al. 2015; Krkovic et al. 2019; Lincoln et al. 2009; Oliver et al. 2012; Thewissen et al. 2011) and theoretical models have stressed anxiety as being particularly relevant to delusion formation (e.g. Freeman et al. 2002) and (4) do not include clinical control groups to examine psychosis-specificity. Thus, we are still unable to answer the question of whether psychosis is associated with a deficit in the ability to apply reappraisal successfully.

Finally, the separate strands of research on the successful use of reappraisal in experimental paradigms and the habitual use of reappraisal reported in questionnaires have hardly been related to each other so far. It seems plausible that if an individual is not able to reduce negative affect via reappraisal, this individual would have little incentive to apply that strategy habitually. Vice versa, a strategy that is seldom used gets little practice, which could impede its effectiveness. Studies examining this association in the context of depression and $\mathrm{HC}$ have yielded equivocal findings (e.g. positive correlations in McRae et al. 2012a, no association in Dillon and Pizzagalli 2013; Wolgast et al. 2011). These findings cannot necessarily be generalized to psychosis because the absence of an association in depression might be driven by motivational aspects (e.g. principally being able to use reappraisal successfully, but not doing so habitually due to a lack of motivation). Even though motivational aspects might also be relevant in PD with negative symptoms, PD may also be more likely to experience difficulties in applying reappraisal successfully (e.g. due to neurocognitive deficits) and thus be less likely to use the strategy habitually. Whether there is such a correlation in PD therefore still needs to be examined.

Summing up, although PD report to use reappraisal to a lesser extent than HC, there is still a significant amount of uncertainty regarding potential deficits in the successful use of reappraisal. Also, it is unknown whether the habitual use of reappraisal is related to its success and 
whether alexithymia, executive functions and a jumpingto-conclusion bias account for group differences regarding reappraisal success. Improving on several design aspects of previous research (e.g. sample size, inclusion of a clinical control group, focus on more than one emotion and assessment of subjective and psychophysiological indicators of emotion-regulation), we hypothesized that: (1a) PD report to use reappraisal less often compared to $\mathrm{HC}$ and patients with anxiety disorders (AD), and (1b) PD are less successful compared to $\mathrm{HC}$ and $\mathrm{AD}$ in reducing experimentally induced anxiety and sadness through reappraisal; (2) the self-reported habitual use of reappraisal is related to whether it can be applied successfully; (3a) alexithymia, (3b) neurocognitive deficits, and (3c) the jumping-to-conclusion bias mediate group differences in reappraisal success.

\section{Methods}

\section{Procedure}

The data was collected in a research project on emotionregulation in psychosis (LI 1298/7-1) that was approved by the Ethical Committee of the German Psychological Society. Within this project, eligible participants (PD, individuals at clinical high risk for psychosis, $\mathrm{AD}$ and $\mathrm{HC}$ ) were invited for a detailed assessment of inclusion criteria, sociodemographic information, clinical data, and habitual emotionregulation skills.

On two additional assessment days, neuropsychological tests were conducted followed by a social exclusion paradigm and an emotion-regulation paradigm that were conducted in a randomized order. All participants were paid for participating in the study. Data related to the social exclusion paradigm (Lincoln et al. 2018; Sundag et al. 2018) and questionnaire data (Ascone et al., 2020) have been published elsewhere. Here, we analyze the data from the questionnaires and from the experimental paradigm on emotion-regulation. We use the data provided by the $\mathrm{PD}, \mathrm{AD}$, and $\mathrm{HC}$ and focus on reappraisal as the strategy of interest.

\section{Participants}

Participants were recruited from inpatient and outpatient treatment settings in and around Hamburg/Germany via online advertisement and advertisements in local newspapers/bulletin boards. Inclusion criteria for all participants were: (1) age between 18 and 65 years, (2) sufficient fluency in the German language, (3) ability to provide informed consent, (4) IQ > 85, (5) no acute suicidality, and (6) no neurological disorders, dementia, diagnosis of bipolar disorder or substance-dependence.
PD fulfilled the criteria for a diagnosis of a psychotic disorder according to the Mini International Neuropsychiatric Interview (M.I.N.I., Sheehan et al. 1998) either (a) acutely or within the previous two years, or (b) at any time of their life, if they had experienced at least two psychotic episodes, were currently taking antipsychotic medicine and scored either at least mildly on two items from the positive or negative subscale of the Positive and Negative Syndrome Scale (PANSS; score $=3$; Kay, Fiszbein, $\&$ Opfer, 1987) or at least moderately on one item (score $\geq 4$ ).

$\mathrm{AD}$ were required to fulfill criteria for at least one anxiety disorder (social phobia, generalized anxiety disorder or panic disorder with or without agoraphobia) in the M.I.N.I. at the time of assessment, but to not fulfill criteria for schizophrenia, schizoaffective, or delusional disorder.

$\mathrm{HC}$ were required to not fulfill criteria for any past or present axis-I disorder in the M.I.N.I., to not be taking medication for any type of mental problem and to not have a firstdegree relative with a psychotic disorder.

\section{Measures}

\section{Psychopathology}

Symptom severity in PD was assessed with the Positive and Negative Syndrome Scale (PANSS; Kay et al. 1987). This semi-structured interview assesses positive symptoms (7 items), negative symptoms (7 items) and global psychopathology (16 items). Internal consistencies for this study are within an acceptable range (positive subscale: $\alpha=.67$; negative subscale: $\alpha=.70$; global psychopathology: $\alpha=.71$ ).

\section{Habitual Reappraisal}

The habitual use of reappraisal was measured with the positive reappraisal subscale of the Cognitive Emotion Regulation Questionnaire (CERQ; German version; Loch et al. 2011), a 36-item questionnaire designed to assess the habitual use of nine separate cognitive emotion-regulation strategies (positive reappraisal, acceptance, positive refocusing, refocus on planning, putting into perspective, self-blame, rumination, catastrophizing, and blaming others). It is rated on a 5-point scale ranging from 1 (almost never) to 5 (almost always). The internal consistency for the positive reappraisal subscale in our study is acceptable $(\alpha=.75)$.

\section{Alexithymia}

Problems in identifying and describing one's emotions were assessed with the German version of the Toronto Alexithymia Scale (TAS-20; Bach et al. 1996). It consists of 20 items that are rated on a 5-point scale ranging from 1 (not 
applicable) to 5 (always applicable). The internal consistency in our study is acceptable $(\alpha=.74)$.

\section{Neurocognitive Functioning}

The Trail-Making-Test (TMT; Reitan 1958) was conducted as an indicator of neurocognitive capacity (processing speed, visual search, scanning, cognitive flexibility, and executive functions). The TMT consists of two parts with TMT-A requiring participants to connect 25 encircled numbers scattered on a sheet of paper in ascending order and TMT-B requiring to connect numbers and letters in an alternative order (e.g. 1-A-2-B-...). The score is equivalent to the time in seconds needed to complete each task, respectively.

\section{Jumping-to-Conclusion Bias (JTC)}

JTC was assessed with the fish-task (Moritz et al. 2010), which is a revised and computerized version of the beads task (Huq et al. 1988). In this task, fish are caught one after another from one of two lakes that are filled with red and gray fish in opposing ratios ( $80 \%$ red and $20 \%$ grey). The participants have to decide from which of the two lakes the fish are caught and are allowed to request seeing as many fish as they like before deciding. There was no limit as to how many fish could be drawn. JTC was operationalized as making a decision after the first fish, as was done by Moritz and colleagues (2010).

\section{Emotion-Regulation Paradigm}

Reappraisal success was assessed with a commonly used experimental emotion-regulation paradigm in which negative affect is induced via affective stimuli and subsequently regulated via instructed emotion-regulation strategies. In this study, pictures from the International Affective Picture System (IAPS; Lang 2005) were used to induce anxiety and sadness (for normative ratings on discrete emotions and arousal see Appendix (Table 3)). During each trial, one of 16 anxiety-evoking and 16 sadness-evoking IAPS-pictures was presented for $9 \mathrm{~s}$ (induction phase, see Fig. 1). This was followed by an intensity-rating of the subjective emotion intensity (t1). Subsequently, an instruction was presented for $3 \mathrm{~s}$ informing the participants which emotion-regulation strategy to use (experimental condition: reappraisal, distraction, or acceptance) or instructing them to just view (control condition) while being presented with the same picture again for $12 \mathrm{~s}$. This was followed by another intensity-rating of the subjective emotion intensity ( $\mathrm{t} 2$ ).

To begin with, participants were asked to sit still for a 4-min baseline heart rate (HR) measurement, and to rate their baseline anxiety and sadness level (t0). Then they completed a 10-min training phase in which instructions on how to apply the different emotion-regulation strategies where depicted on the screen followed by four anxiety eliciting pictures to practice each strategy and the control condition (just view). For reappraisal, the participants received the following instruction: "Look at the picture attentively. Try to change the emerging emotions by reappraising the situation".
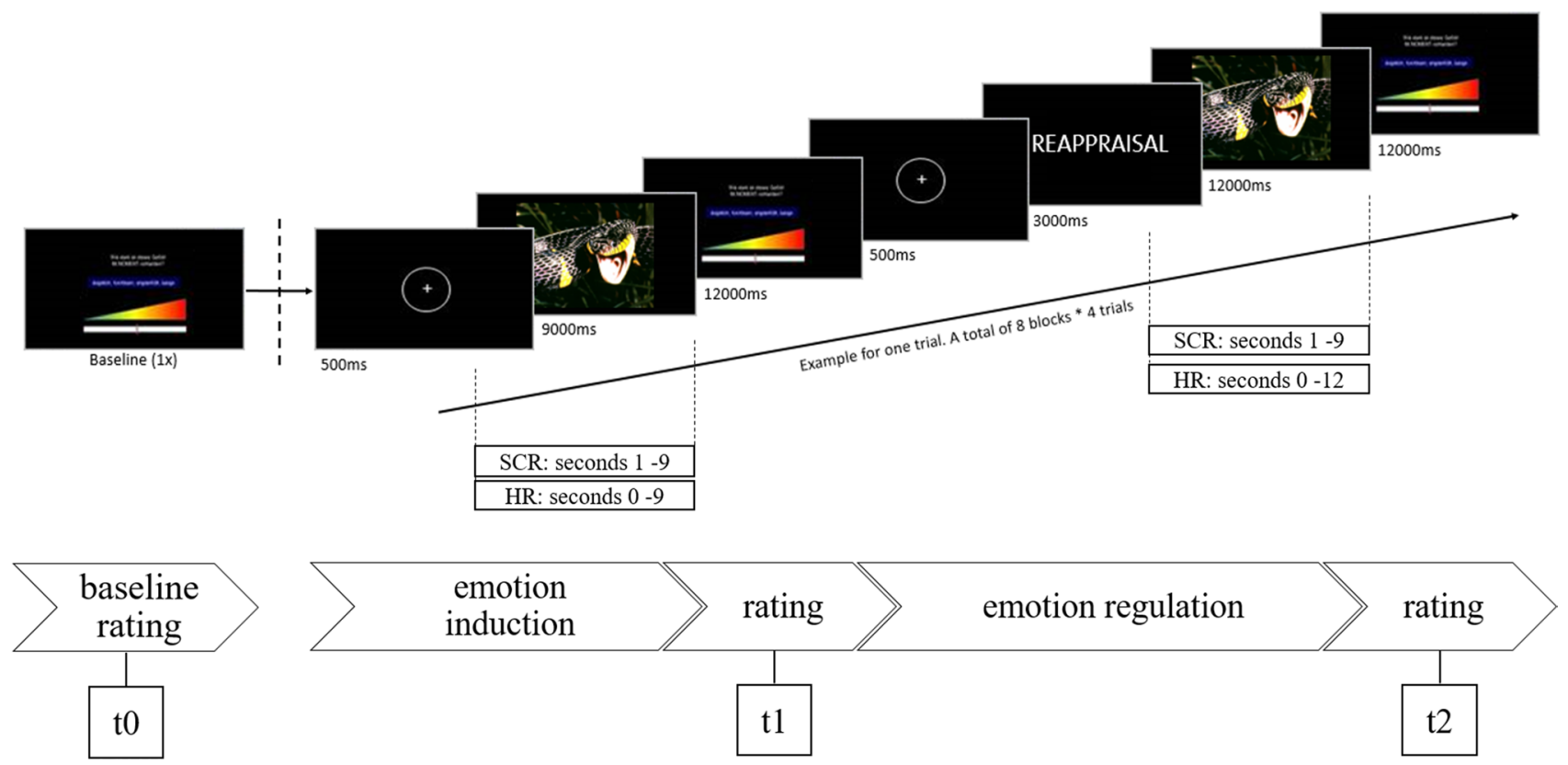

Fig. 1 Structure of a trail within the experimental emotion-regulation paradigm (Color figure online) 
For the control condition (view), participants received the following instruction: "Look at the picture attentively. Concentrate on what is depicted on the picture. Do not apply another strategy (reappraisal, distraction, acceptance)". This was followed by the experimental paradigm, which was constructed as a randomized block design with each block consisting of four trials. The emotion-regulation paradigm was conducted as $4 \times 2 \times 4$ mixed experimental design with emotion-regulation strategies (reappraisal, distraction, acceptance, view) and emotion (anxiety and sadness) as within subject factors and group as the between subject factor (PD, $\mathrm{AD}$, individuals at clinical high risk for psychosis and $\mathrm{HC}$ ). For the purpose of this study, we only used the reappraisal and the view condition.

The dependent variables were picture-congruent emotion intensity and physiological arousal. Emotion intensity was rated by the participant after each picture on well-validated 11-point rating scales $(1=$ "not applicable", $11=$ "applicable"; Stemmler et al. 2001). The scales were labeled with four adjectives selected to capture anxiety (frightened/ timid/ afraid/ scared) and sadness (sad/ depressed/ miserable/ dejected).

Physiological arousal, indicated by the skin conductance and HR, was recorded continuously during the experiment. The skin conductance was measured with a sampling rate of $32 \mathrm{~Hz}$ using the NeXus $32^{\mathrm{TM}}$ BioTrace ${ }^{\mathrm{TM}}$ system by Mind Media. $\mathrm{Ag} / \mathrm{AgCl}$ electrodes were placed at the distal phalanx of the second and third finger of the left hand. The skin conductance data was processed using Matlab R2020a and Ledalab V3.4.9. Data was downsampled to $16 \mathrm{~Hz}$ and the minimum amplitude threshold was set to $0.01 \mu \mathrm{S}$. We used continuous decomposition analysis (Benedek and Kaernbach 2010) to extract the continuous phasic activity. For each trial we calculated the 'area under the curve' in terms of the integrated skin conductance response (ISCR; measured in $\left.\mu \mathrm{S}^{*} \mathrm{sec}\right)$ as a representation of the "strength of affect" (Boucsein, 2012, p.165) separately for the emotion induction and the regulation phases. The time-frame of the analysis was $8 \mathrm{~s}$, starting at $1 \mathrm{~s}$ after stimulus presentation, respectively, due to latency effects of the skin conductance response. ISCR-values were additionally log-transformed to obtain normal distribution. Participants were excluded from the analyses of skin conductance data in the case of insufficient quality/missing data and regular (=daily) substance abuse. The NeXus 32 $2^{\mathrm{TM}}$ BioTrace ${ }^{\mathrm{TM}}$ system was also used to record HR. The blood volume pulse was measured from the index finger of the non-dominant hand with a sampling rate of 256/s. Visual artefact screening was performed. HR was averaged across $9 \mathrm{~s}$ in the induction phase and $12 \mathrm{~s}$ in the regulation phase. HR-values were additionally logtransformed to obtain normal distribution. Participants were excluded from the analyses of HR data in the case of insufficient quality/missing data, cardiovascular disease or anomalies or cardiac medication (e.g. beta-blockers, angiotensin-converting enzyme inhibitors). Participants stayed connected to the psychophysiological sensors throughout the entire emotion-regulation paradigm.

\section{Statistical Analyses}

Analyses were conducted in SPSS (version 24). To test for group differences in sample characteristics and habitual use of reappraisal, one-way analyses of variance (ANOVAs) and $\chi^{2}$-tests were conducted, respectively. Where necessary due to a partial violation of assumptions, analyses were repeated with non-parametric tests (Kruskal-Wallis). To test for group differences in reappraisal success, separate mixed repeated measures ANOVAs were conducted for each dependent variable (subjective picture-congruent emotion intensity, ISCR and HR). Type of stimuli (anxiety vs. sadness inducing IAPS picture), timepoint (baseline, preregulation, post-regulation; for event-related ISCR only preregulation and post-regulation) and condition (reappraisal vs. view) were entered as within-subject factors and group (PD vs. AD vs. HC) was entered as the between-subject factor. The Greenhouse-Geisser adjustment was used to correct for violations of sphericity. Bonferroni adjustment was applied for post-hoc $t$-tests.

We expected the following effects: (1) a significant main effect for time with post-hoc tests showing a significant increase of picture-congruent emotion intensity and HR from baseline (t0) to post emotion induction (t1) across all groups, both conditions and both emotions (manipulation check); (2) a significant time*condition interaction with post-hoc tests showing no differences between conditions at baseline ( $\mathrm{t} 0$ ) or after emotion induction ( $\mathrm{t} 1$ ), but a significantly lower emotion intensity after reappraisal than after viewing (t2); (3) a significant time*condition*group interaction effect, indicating that the time*condition interaction effect described previously differs between groups. Specifically, we expected post-hoc tests to show a significantly higher emotion intensity after reappraisal in PD compared to $\mathrm{HC}$ and $\mathrm{AD}$. In addition, we explored whether the type of emotion had an effect on potential reappraisal deficits in PD by also testing for a time*condition*group*emotion interaction.

In the initial analyses of the psychophysiological data (ISCR and HR), age, gender and cigarettes smoked per day were considered as covariates due to their potential effects on autonomic activity. Since no covariate reached significance, the final analyses were conducted without covariates.

Pearson correlation was used to test the interrelation between habitual use of reappraisal and reappraisal success (subjective success: difference in state anxiety and sadness from $t 1$ to 2 within the reappraisal condition; physiological 
indicators of reappraisal success: difference in ISCR and HR from $\mathrm{t} 1$ to $\mathrm{t} 2$ within the reappraisal condition).

\section{Results}

\section{Sample Characteristics}

Within the PD sample $(n=60)$, diagnoses included schizophrenia $(n=41)$, schizoaffective disorder $(n=15)$, delusional disorder $(n=3)$, and brief psychotic disorder $(n=1)$. The majority of PD $(n=57)$ received antipsychotic medication (chlorpromazine equivalence dose of $M=437.60$, $S D=483.86$, calculated as proposed by Benkert and Hippius 2006). Within the AD sample $(n=40)$, disorders included social anxiety $(n=13)$, panic disorder with/without agoraphobia $(n=30)$, and generalized anxiety disorder $(n=10)$. Two AD reported to take antipsychotic medication (chlorpromazine equivalence dose of $M=8.33, S D=11.79)$. PD did not differ from AD and HC regarding age, gender distribution and years of education. See Table 1 for means and statistics.

\section{Group Differences in the Habitual Use of Reappraisal}

The one-way ANOVA revealed significant group differences in habitual reappraisal, $F(2,137)=8.085, p<0.001$; $\eta_{\text {partial }}^{2}=0.106$. Contrary to our hypothesis, pairwisecomparisons showed no significant difference in habitual reappraisal between PD and HC $(p=0.099)$. However, PD reported to use reappraisal significantly more often than $\mathrm{AD}$ $(p=0.008)$. Additionally, AD reported to use reappraisal significantly less often than HC $(p<0.001)$. See Table 2 for group-means and standard deviations.

\section{Group Difference in Reappraisal Success}

Results of reappraisal success for all outcome variables (emotion intensity, ISCR and HR) and both experimental conditions (reappraisal vs. view) are visualized in Figs. 2, 3 and 4 . The following report is restricted to the effects that were hypothesized. An account of all effects is provided in the Appendix (Tables 4, 5, 6).

Table 1 Sample characteristics and between-group differences

\begin{tabular}{lllllll}
\hline Variable & Group & Parametric tests & Non-parametric tests \\
\cline { 2 - 5 } & $\begin{array}{l}\text { Patients with psychotic } \\
\text { disorders } n=60 M(S D)\end{array}$ & $\begin{array}{l}\text { Patients with anxiety } \\
\text { disorders } n=40 M \\
(S D)\end{array}$ & $\begin{array}{l}\text { Healthy control } \\
\text { subjects } n=40 M \\
(S D)\end{array}$ & & \\
\hline Age in years & $40.15(11.66)$ & $42.23(11.64)$ & $40.03(10.78)$ & & $F(2,137)=.497, p=.609$ & $H(2)=1.476, p=.478$ \\
Gender (\%female) & 63.3 & 62.5 & 67.5 & - & $\chi^{2}(2)=.26, p=.878$ \\
Years of education & $11.71(1.57)$ & $11.33(1.72)$ & $11.86(1.53)$ & & $F(2,137)=1.224, p=.297$ & $H(2)=2.091, p=.352$ \\
PANSS positive & $15.38(5.28)$ & - & - & & \\
PANSS negative & $11.98(4.90)$ & - & - & & \\
PANSS general & $30.45(7.57)$ & - & - & & \\
\hline
\end{tabular}

Education refers to school education, without academic degrees

PANSS Positive and Negative Syndrome Scale (positive symptom subscale, negative symptom subscale, and general psychopathology subscale)

Table 2 Between-group differences in self-reported habitual reappraisal use, alexithymia, jumping-to-conclusion and neurocognition

\begin{tabular}{|c|c|c|c|c|c|}
\hline \multirow[t]{2}{*}{ Variable } & \multicolumn{3}{|l|}{ Group } & \multirow[t]{2}{*}{ Parametric tests } & \multirow[t]{2}{*}{ Non-parametric tests } \\
\hline & $\begin{array}{l}\text { Patients with psy- } \\
\text { chotic disorders } M \\
(S D)\end{array}$ & $\begin{array}{l}\text { Patients with anxiety } \\
\text { disorders } M(S D)\end{array}$ & $\begin{array}{l}\text { Healthy control } \\
\text { subjects } M(S D)\end{array}$ & & \\
\hline CERQ reappraisal & $8.83(3.03)$ & $7.23(2.58)$ & $9.83(3.07)$ & $F(2,137)=8.085, p<.001$ & $H(2)=14.566, p=.001$ \\
\hline TAS & $51.25(10.35)$ & $54.68(11.15)$ & $39.78(8.01)$ & $F(2,137)=25.031, p<.001$ & $H(2)=39.365, p<.001$ \\
\hline JTC bias (\% yes) & 41.7 & 37.5 & 32.5 & - & $\chi^{2}(2)=0.86, p=.650$ \\
\hline TMT A & $31.21(20.05)$ & $27.25(10.59)$ & $22.43(5.69)$ & $F(2,137)=4.334, p=.015$ & $H(2)=8.426, p=.015$ \\
\hline TMT B & $61.32(24.61)$ & $54.22(19.85)$ & $44.16(13.51)$ & $F(2,135)=8.259, p<.001$ & $H(2)=13.048, p=.001$ \\
\hline
\end{tabular}

CERQ Cognitive Emotion Regulation Questionnaire (positive reappraisal subscale), TAS Toronto Alexithymia Scale, JTC jumping-to-conclusion bias measured with the fish-test, TMT Trail Making Test (version A and B) 
REAPPRAISAL

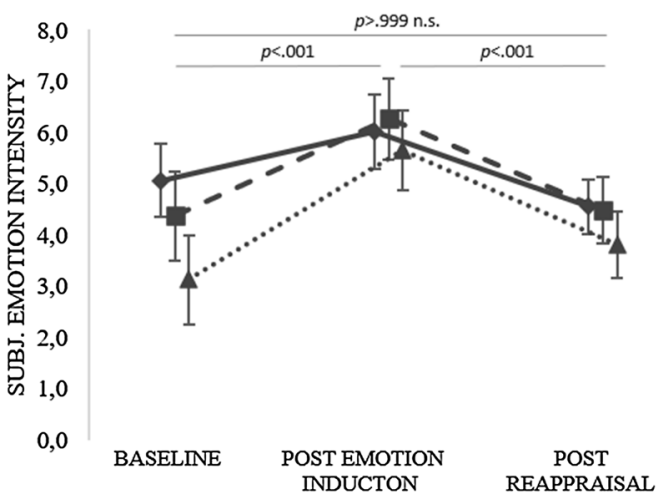

VIEW

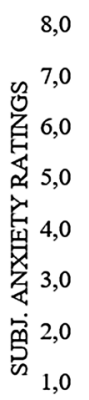

0,0

BASELINE

$p<.001$
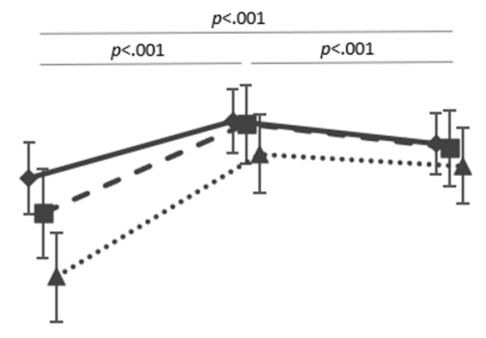

patients with psychosis

- E p patients with anxiety

disorders

....... healthy controls

Fig. 2 Graphical display of subjective emotion intensity within the reappraisal and control condition

REAPPRAISAL

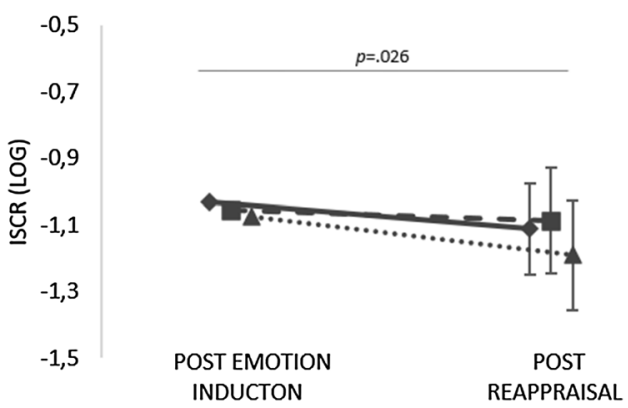

VIEW

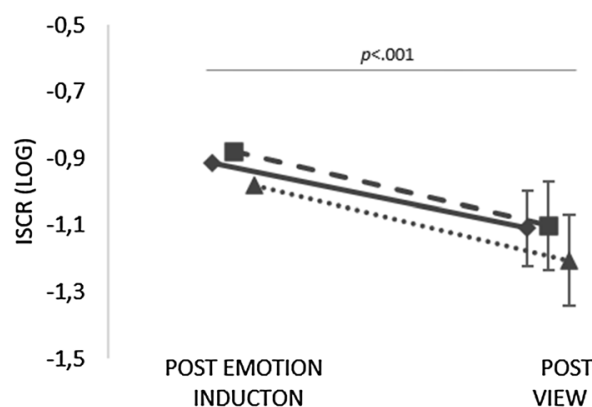

Fig. 3 Graphical display of integrated skin conductance response (ISCR; time intergral of phasic driver over response window; logtransformed) within the reappraisal and control condition. After applying the additional exclusion criteria for the psychological data, the samples comprised $n=128$ for the ISCR-analyses (PD: $n=53$; AD: $\mathrm{n}=39$; HC: $n=36$ )
REAPPRAISAL

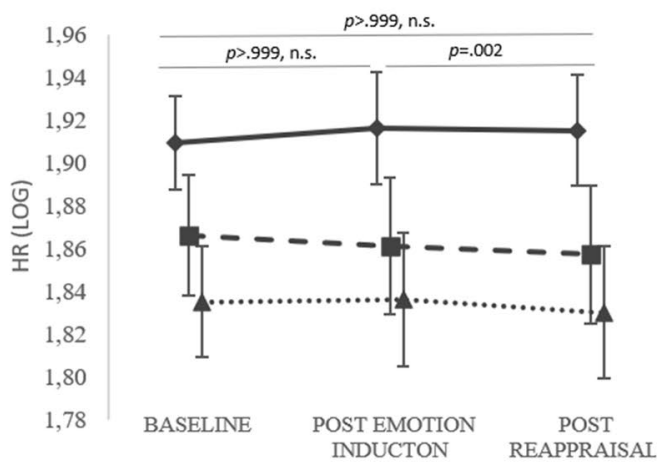

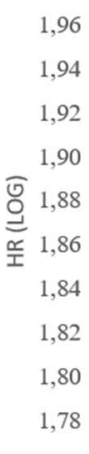

\section{VIEW}

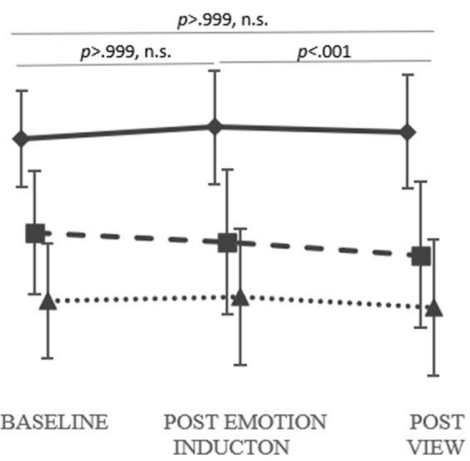

ـ patients with psychosis

- - patients with anxiety disorders

$\cdots$..... healthy controls

Fig. 4 Graphical display of heart rate (HR; log-transformed) within the reappraisal and control condition. After applying the additional exclusion for the psychological data, the samples comprised $n=103$ for the HR-analyses (PD: $n=44$, AD: $n=28$, HC: $n=31$ ) 


\section{Subjective Success}

For the self-report of emotion intensity, the within-subject analysis revealed a significant main effect for time, $F(1.238$, $169.562)=42.993, p<0.001, \eta_{\text {partial }}^{2}=0.239$, with post hoc-tests showing a significant increase of picture-congruent emotion intensity from to to $\mathrm{t} 1$ across all groups, conditions and types of emotion elicited $(p<0.001)$, which indicates that emotion was successfully manipulated by the IAPS pictures. Negative emotions decreased from $\mathrm{t} 1$ to $\mathrm{t} 2$ $(p<0.001)$ but remained significantly higher at $\mathrm{t} 2$ compared to t0 $(p=0.004)$. A significant interaction effect was found for time*condition, $F(1.655,226.667)=77.973, p<0.001$, $\eta_{\text {partial }}^{2}=0.363$ with post-hoc tests indicating no differences in emotion intensity ratings between the conditions at $t 0$ and t1 (both $p s>0.05$ ), but significant differences between conditions at $\mathrm{t} 2$ with lower levels of emotion intensity in the reappraisal condition compared to the view condition $(p<0.001)$. Within the reappraisal condition, emotion intensity at $\mathrm{t} 2$ did not differ from t0 $(p>0.999)$. Within the viewing condition, emotion intensity at $\mathrm{t} 2$ was significantly higher compared to t0 $(p<0.001)$. No significant interaction effect was found for condition*time*group, $F(3.309$, $226.667)=1.229, p=0.300, \eta_{\text {partial }}^{2}=0.018$, indicating that PD showed a reduction of emotion intensity after reappraisal that was comparable to the reductions in both control groups. Finally, no significant interaction effect was found for condition*time*group*emotion, $F(3.701,253.503)=1.225$, $p=0.301, \eta_{\text {partial }}^{2}=0.018$.

\section{Physiological Success}

For ISCR as dependent variable, the within-subject analysis revealed a significant main effect for time, $F(1$, $125)=43.136, p<0.001, \eta_{\text {partial }}^{2}=0.257$, with post hoctests showing a significant decrease from t1 to t $2(p<0.001)$. There was a significant interaction effect for time*condition, $F(1,125)=11.553, p=0.001, \eta_{\text {partial }}^{2}=0.085$, with post hoc-tests showing a higher ISCR within the view compared to the reappraisal condition at $\mathrm{t} 1(p<0.001)$, but not at $\mathrm{t} 2$ $(p=0.769)$. ISCR reduced from $\mathrm{t} 1$ to $\mathrm{t} 2$ significantly in both the view condition $(p<0.001)$ and the reappraisal condition $(p=0.026)$. There was no significant interaction effect for condition*time* group, $F(2,125)=0.423, p=0.656$, $\eta_{\text {partial }}^{2}=0.007$, or for condition*time* group*emotion, $F(2$, $125)=0.124, p=0.884, \eta_{\text {partial }}^{2}=0.002$.

For HR as a dependent variable, the within-subject analysis revealed no significant main effect for time, $F(1.022$, $102.244)=0.271, p=0.609, \eta_{\text {partial }}^{2}=0.003$. There was no significant interaction effect for time*condition, $F(2,200)=0.296, p=0.744, \eta^{2}$ partial $=0.003$, for condition*time*group, $F(3.992,199.577)=1.092, p=0.362$, $\eta_{\text {partial }}^{2}=0.021$, or for condition*emotion*time*group, $F(3.818,190.885)=1.491, p=0.209, \eta_{\text {partial }}^{2}=0.029$.

\section{Interrelation of Habitual Reappraisal Use and Reappraisal Success}

The correlation between habitual reappraisal use and subjective reappraisal success across all groups and both types of emotion elicited was $r=0.087, p=0.309$ and remained non-significant when calculated per group (PD: $r=0.108, p=0.410$; AD: $r=0.036, p=0.828$; HC: $r=0.111$, $p=0.496)$. Also, no significant correlations were found between the habitual reappraisal use and physiological indicators of reappraisal success, neither for ISCR (across all groups: $r=-0.012, p=0.892$;D: $r=0.022, p=0.872$; AD: $r=-0.247, p=0.130$; HC: $r=-0.006, p=0.972)$ nor for HR (across all groups: $r=-0.122, p=0.163$; PD: $r=-0.079, p=0.558$; AD: $r=-0.117, p=0.473$; HC: $r=-0.093, p=0.589$ ).

\section{Alexithymia, Cognitive Functioning and Jumping-to-Conclusion Bias as Mediators}

As can be seen in Table 2, one-way ANOVAs revealed significant group differences for emotion awareness and TMT-A and TMT-B performance. Pairwise comparisons indicated that $\mathrm{PD}$ and $\mathrm{AD}$ reported more difficulties with emotion awareness than HC (both $p$ ` $s<0.001$ ), whereas PD and AD did not differ $(p=0.286)$. Pairwise comparisons indicated poorer TMT performance in PD compared to HC (TMT-A: $p=0.012$; TMT-B: $p<0.001$ ), but not compared to AD (TMT-A: $p=0.564$; TMT-B: $p=0.285$ ). No group differences were found for JTC.

The planned mediation analyses were discarded due to lack of group differences in reappraisal success and because there were no significant correlations between reappraisal success and alexithymia, cognitive functioning or JTC, respectively (all $p ` s>0.005$ ).

\section{Discussion}

The main focus of this study was to examine potential psychosis-specific deficits in the habitual use and the successful application of reappraisal.

Our hypothesis that PD would report to use less reappraisal than $\mathrm{HC}$ and $\mathrm{AD}$ was not supported by our data. Although our study is unlikely to fundamentally change the small to moderate effect in a recent meta-analysis that indicated less habitual reappraisal in PD (Ludwig et al. 2019), it adds to the existing heterogeneity. This raises the question of possible moderators. Differences in patient characteristics, such as the amount of psychological therapy or acuteness of 
disorder might contribute to the differences found between studies and should be considered in further research. Interestingly, several recent experience sampling studies even indicate a higher use of reappraisal in PD compared to $\mathrm{HC}$ (Ludwig et al. 2020; Visser et al. 2018). We would argue that the findings from these studies are more reliable than the retrospective questionnaire assessments because they enable a timely assessment of the use of reappraisal in daily life and are less susceptible to recall biases. Thus, we may have been underestimating the habitual use of reappraisal in psychosis.

Regarding regulation success, our results indicate that $\mathrm{PD}$ are as successful as $\mathrm{HC}$ and $\mathrm{AD}$ in reducing subjective anxiety and sadness through reappraisal. Even though this was also unexpected, it corroborates several other studies finding no deficits in subjective reappraisal success in psychosis (Grezellschak et al. 2015; Painter et al. 2019; Perry et al. 2012). Since the symptomatology in our sample is comparable to other samples of PD seeking therapeutic treatment (e.g. Lincoln et al. 2012; Mehl et al. 2018), it is unlikely that the lack of deficits is explicable by having drawn a particularly healthy sample. Regarding the physiological indicators, we found a reduction of the skin conductance response in both the reappraisal and the control condition. The fact that these results do not mirror the results of the subjective ratings in all aspects is in line with other studies reporting discrepancies between self-reported and physiological arousal in psychosis (Söder et al. 2018). The failure to find an effect of reappraisal on HR and the skin conductance response is in line with a previous study (Westermann et al. 2014). PD showed a significantly higher HR than AD and HC across all timepoints [see Appendix (Table 6)], a pattern which has also been found in other studies (e.g. Bär et al. 2005; Lincoln et al. 2015; Rechlin et al.1994; Valkonen-Korhonen et al. 2003). Following emotional stimuli, an initial HR deceleration can be observed as an orienting response followed by a HR acceleration (Bradley 2009). Because we aimed to assess $\mathrm{HR}$ as a physiological indicator of ER success rather than to examine the emotional response itself, we examined the mean HR. This approach was also chosen in similar studies (e.g. Painter et al. 2019; Westermann et al. 2014). However, since HR did not increase in response to the emotioneliciting images, it is difficult to interpret this measure as an indicator for reappraisal success.

Taken together, our study suggests that people with psychotic disorders can successfully use reappraisal, at least when they are instructed to do so in a laboratory setting. This does not rule out the possibility that the distress resulting from individually relevant and potentially psychosisspecific triggers in daily life might be more difficult to regulate. However, recent findings from an experience sampling study indicate intact reappraisal abilities in PD outside of the laboratory (Ludwig et al. 2020). Taken together, a growing number of studies do not support the notion that psychosis is associated with a deficit in using reappraisal successfully when operationalized in terms of subjective emotion experience. Nevertheless, studies using fMRI or EEG found differences between PD and $\mathrm{HC}$ in the modulation of neural responses (late positive potentials) through reappraisal (Horan et al. 2013; Strauss et al. 2013), and in the activation of brain regions associated with emotion-regulation (Modinos et al. 2010; Van Meer et al. 2014) that point towards regulation deficits within the neural circuits. The reasons for this discrepancy need to be further elucidated. Possibly, reappraisal deficits that are detectable on the neural level may be overridden by other processes (e.g. by compensatory mechanisms; Modinos et al. 2010; Phillips and Seidman 2008) and therefore do not translate into subjective experience, or a lack of emotion awareness contributes to the discrepancies between subjective report and physiological measures, as has been suggested in other studies (Söder et al 2018). Another possibility concerns the time point when reappraisal is applied: two of the studies that found differences between PD and HC in the late positive potentials examined reappraisal as a antecedent-focused strategy by preceding emotion eliciting pictures with either negative or neutral descriptions (Horan et al. 2013; Strauss et al. 2013). In contrast, all studies focusing on the subjective success (including ours) asked participants to apply reappraisal during or after emotion generation, thus examining reappraisal as a response-focused strategy. Whether the timing of reappraisal might have an effect on regulation success has to be examined in future studies.

Surprisingly, self-reported use of habitual reappraisal and the ability to apply this strategy successfully were uncorrelated. As non-significance was found within each group, the missing association is unlikely to be due to disorder specific factors such as higher alexithymia in PD. Alternative explanations are that questionnaire-based estimates of habitual reappraisal are inaccurate, or that reappraisal success seen in a laboratory setting is not equivalent to reappraisal success in daily life. In any case, the missing association suggests that we cannot generalize findings of reduced habitual use of reappraisal from questionnaire-based studies to surmise general deficits in the use of that strategy. Rather, we may need to distinguish between different forms and levels on which deficits might occur, such as the frequency in the habitual use, the success in reducing negative affect on a subjective or a physiological level, or the ability to select an appropriate strategy depending on the context (see also McRae 2013).

Finally, our mediation hypotheses were also not supported because we did not find differences in reappraisal success between groups or correlations between reappraisal success and alexithymia, neurocognition and jumping-to-conclusion biases, respectively. However, a recent study suggests that neurocognition might be more relevant when subjects have 
to flexibly choose emotion-regulation strategies than when they implement instructed strategies (Pruessner et al. 2020).

\section{Limitations and Suggestions for Further Research}

When interpreting the results on regulation success, it has to be considered that the subjective emotion intensity ratings might have been influenced either by social desirability or by expectancy effects, which is a general limitation of subjective measures. This is particularly likely because the participants were aware that they performed emotion regulation, which is difficult to mask in such experiments. Thus, those biases might have contributed to the reduction in subjectively reported negative affect we see in all groups following reappraisal. The possibility of such biases emphasizes the need to also consider physiological indicators that are less likely to be biased than subjective ratings. As we did not see group specific differences in the physiological indicators over time (no time*condition*group interactions), there is no reason to assume that social desirability or expectancy effects affected the ratings of PD specifically. Thus, they are unlikely to have had an impact on our main research question.

Even though all participants received specific instruction on which emotion-regulation strategy to use (or not to regulate), we do not know whether and to which extent those instructions were followed, and we cannot rule out that additional strategies were used. Future studies might benefit from either including specific questions to query whether instructions were followed or from instructing participants to think aloud while applying the strategies.

\section{Conclusion and Therapeutic Implications}

The prevalence of high levels of negative affect in PD points to some type of deficit in emotion-regulation in this patient population. However, contrary to the common assumption and despite prevalent problems with alexithymia and neurocognitive functioning, this study found no deficits in PD regarding the habitual and successful use of reappraisal after instruction. Nevertheless, it remains unclear, whether PD are able to use reappraisal successfully without instruction, whether they are able to select this strategy in appropriate situations and why most studies report that they use reappraisal less habitually. Those questions need to be addressed before disregarding the relevance of reappraisal in psychosis altogether. From a clinical perspective, the finding that PD are able to use reappraisal successfully after instruction and in controlled conditions is promising, because it suggests that they could be guided by therapists on how to use it successfully in everyday life.

Author Contributions TL and JS contributed to the study conception and design. JS performed material preparation and data collection. SO performed analyses and wrote the first draft of the manuscript. MR has contributed substantially to the analyses of the skin conductance data. All authors commented on previous versions of the manuscript. All authors read and approved the final manuscript.

Funding Open Access funding enabled and organized by Projekt DEAL. This work was supported by the German Research Foundation (Grant No. DFG Li 1298/7-1).

\section{Compliance with Ethical Standards}

Conflict of Interest Sandra Opoka, Johanna Sundag, Marcel Riehle and Tania Lincoln declare that they have no conflict of interest.

Ethical Approval All procedures were in accordance with the ethical standards of the Ethical Committee of the German Psychological Society and with the 1964 Helsinki declaration and its later amendments.

Informed Consent All participants provided written informed consent.

Animal Rights No animal studies were carried out by the authors for this article.

Open Access This article is licensed under a Creative Commons Attribution 4.0 International License, which permits use, sharing, adaptation, distribution and reproduction in any medium or format, as long as you give appropriate credit to the original author(s) and the source, provide a link to the Creative Commons licence, and indicate if changes were made. The images or other third party material in this article are included in the article's Creative Commons licence, unless indicated otherwise in a credit line to the material. If material is not included in the article's Creative Commons licence and your intended use is not permitted by statutory regulation or exceeds the permitted use, you will need to obtain permission directly from the copyright holder. To view a copy of this licence, visit http://creativecommons.org/licenses/by/4.0/.

\section{Appendices}

\section{Appendix A}

See Table 3 
Table 3 Selected IAPS pictures with ratings for sadness, anxiety and arousal

\begin{tabular}{|c|c|c|c|c|c|c|}
\hline \multirow[t]{2}{*}{ IAPS No } & \multicolumn{2}{|c|}{ Sadness } & \multicolumn{2}{|c|}{ Anxiety } & \multicolumn{2}{|c|}{ Arousal } \\
\hline & $M$ & $S D$ & $M$ & $S D$ & $M$ & $S D$ \\
\hline \multicolumn{7}{|l|}{ Anxiety } \\
\hline 1022 & 1.96 & 1.61 & 5.31 & 2.74 & 3.73 & 2.38 \\
\hline 1052 & 1.73 & 1.30 & 6.18 & 3.21 & 4.88 & 2.63 \\
\hline 1301 & 2.96 & 2.36 & 5.30 & 2.91 & 3.96 & 2.72 \\
\hline 1930 & 2.81 & 2.30 & 6.04 & 2.63 & 4.85 & 2.62 \\
\hline 1931 & 2.16 & 1.67 & 5.72 & 2.29 & 5.22 & 2.50 \\
\hline 5972 & 5.33 & 2.53 & 6.37 & 2.45 & 4.56 & 2.94 \\
\hline 1120 & 2.41 & 1.89 & 6.41 & 2.66 & 4.30 & 2.73 \\
\hline 1019 & 3.28 & 2.41 & 3.13 & 2.48 & 3.53 & 2.29 \\
\hline 1050 & 2.15 & 1.96 & 6.00 & 2.78 & 4.35 & 2.54 \\
\hline 1051 & 2.23 & 1.90 & 5.23 & 2.83 & 3.88 & 2.41 \\
\hline 1120 & 2.14 & 1.89 & 6.41 & 2.66 & 4.30 & 2.73 \\
\hline 1200 & 1.08 & 0.28 & 5.60 & 2.69 & 3.24 & 2.50 \\
\hline 1201 & 2.08 & 1.99 & 6.39 & 2.73 & 4.76 & 2.90 \\
\hline 1220 & 1.73 & 1.25 & 5.27 & 2.75 & 3.68 & 2.50 \\
\hline 1300 & 2.52 & 1.71 & 5.96 & 2.42 & 3.80 & 2.31 \\
\hline 6370 & 3.81 & 2.58 & 6.65 & 2.42 & 4.32 & 2.90 \\
\hline \multicolumn{7}{|l|}{ Sadness } \\
\hline 2053 & 5.92 & 2.72 & 3.25 & 2.56 & 5.26 & 2.47 \\
\hline 2141 & 7.00 & 2.47 & 4.16 & 2.67 & 3.16 & 2.17 \\
\hline 2205 & 7.38 & 1.84 & 3.41 & 2.63 & 3.67 & 2.46 \\
\hline 2276 & 6.60 & 2.27 & 2.32 & 1.86 & 3.00 & 2.16 \\
\hline 2800 & 7.63 & 1.66 & 2.96 & 2.42 & 5.79 & 2.54 \\
\hline 2900 & 6.35 & 2.35 & 2.81 & 2.61 & 3.62 & 2.29 \\
\hline 3220 & 6.71 & 2.08 & 3.50 & 2.55 & 4.44 & 2.45 \\
\hline 3230 & 6.97 & 2.37 & 4.08 & 2.53 & 3.90 & 2.58 \\
\hline 3300 & 6.29 & 2.39 & 2.58 & 2.26 & 3.95 & 2.43 \\
\hline 3301 & 6.85 & 2.36 & 3.85 & 2.66 & 2.81 & 2.65 \\
\hline 6570 & 6.23 & 2.55 & 3.88 & 2.85 & 5.21 & 2.94 \\
\hline 9041 & 6.08 & 2.16 & 2.44 & 2.06 & 2.84 & 1.99 \\
\hline 9252 & 6.08 & 2.12 & 3.80 & 3.01 & 3.56 & 2.40 \\
\hline 9421 & 7.16 & 2.01 & 6.04 & 2.77 & 3.60 & 2.65 \\
\hline 9561 & 7.49 & 1.78 & 2.04 & 2.01 & 3.84 & 2.45 \\
\hline 9921 & 6.82 & 2.49 & 5.62 & 2.56 & 4.80 & 2.85 \\
\hline
\end{tabular}

Selected from https://psychonomic.org/ archive/. Ratings from Libkuman et al. (2007) 


\section{Appendix B}

See Table 4

Table 4 Statistics for mixed repeated measures ANOVA with subjective emotion intensity as the dependent variable

\begin{tabular}{lllll}
\hline Effects & df & $F$ & $p$ & $\eta$ 2partial \\
\hline Main effects & & & & \\
Condition & 1.000 & 36.499 & $<.001$ & 0.210 \\
Emotion & 1.000 & 21.049 & $<.001$ & 0.133 \\
Time & 1.238 & 42.993 & $<.001$ & 0.239 \\
Group & 2 & 2.893 & .059 & 0.041 \\
Interaction effects & & & & \\
Condition*group & 2.000 & 0.304 & .738 & 0.004 \\
Emotion*group & 2.000 & 1.012 & .366 & 0.015 \\
Time*group & 2.475 & 3.121 & $\mathbf{. 0 3 6}$ & 0.044 \\
Condition*emotion & 1.000 & 0.401 & .528 & 0.003 \\
Condition*emotion*group & 2.000 & 0.423 & .656 & 0.006 \\
Condition*time & 1.655 & 77.973 & $<.001$ & 0.363 \\
Condition*time*group & 3.309 & 1.229 & .300 & 0.018 \\
Emotion*time & 1.221 & 22.079 & $<.001$ & 0.139 \\
Emotion*time*group & 2.443 & 1.568 & .206 & 0.022 \\
Condition*emotion*time & 1.850 & 1.422 & .243 & 0.010 \\
Condition*emotion*time*group & 3.701 & 1.225 & .301 & 0.018 \\
\hline
\end{tabular}

\section{Appendix C}

See Table 5

Table 5 Statistics for mixed repeated measures ANOVA with eventrelated skin conductance response (ISCR) as the dependent variable

\begin{tabular}{lllll}
\hline Effects & df & $F$ & $p$ & $\eta 2$ partial \\
\hline Main effects & & & & \\
Condition & 1 & 6.361 & $\mathbf{. 0 1 3}$ & 0.048 \\
Emotion & 1 & 2.610 & .109 & 0.020 \\
Time & 1 & 43.136 & $\mathbf{< . 0 0 1}$ & 0.257 \\
Group & 2 & 0.485 & .617 & 0.008 \\
Interaction effects & & & & \\
Condition*group & 2 & 0.219 & .804 & 0.003 \\
Emotion*group & 2 & 1.813 & .167 & 0.028 \\
Time*group & 2 & 0.289 & .750 & 0.005 \\
Condition*emotion & 1 & 5.693 & $\mathbf{. 0 1 9}$ & 0.044 \\
Condition*emotion*group & 2 & 0.043 & .958 & 0.001 \\
Condition*time & 1 & 11.553 & $\mathbf{. 0 0 1}$ & 0.085 \\
Condition*time*group & 2 & 0.423 & .656 & 0.007 \\
Emotion*time & 1 & 1.290 & .258 & 0.010 \\
Emotion*time*group & 2 & 0.257 & .774 & 0.004 \\
Condition*emotion*time & 1 & 6.152 & $\mathbf{. 0 1 4}$ & 0.047 \\
Condition*emotion*time*group & 2 & 0.124 & .884 & 0.002 \\
\hline
\end{tabular}

After applying the additional exclusion criteria for the skin conductance-analyses, the sample comprised $n=128$ (PD: $n=53$, AD: $n=39$, HC: $n=36$ )

\section{Appendix D}

See Table 6

Table 6 Statistics for mixed repeated measures ANOVA with heart rate as the dependent variable

\begin{tabular}{lllll}
\hline Effects & df & $F$ & $p$ & $\eta 2$ partial \\
\hline Main effects & & & & \\
Condition & 1.000 & 1.134 & .715 & 0.001 \\
Emotion & 1.000 & 0.227 & .635 & 0.002 \\
Time & 1.022 & 0.271 & .609 & 0.003 \\
Group & 2 & 10.045 & $<.001$ & 0.167 \\
Interaction effects & & & & \\
Condition*group & 2.000 & 1.085 & .342 & 0.021 \\
Emotion*group & 2.000 & 0.606 & .547 & 0.012 \\
Time*group & 2.045 & 0.319 & .733 & 0.006 \\
Condition*emotion & 1.000 & 0.911 & .342 & 0.009 \\
Condition*emotion*group & 2.000 & 2.391 & .097 & 0.046 \\
Condition*time & 2 & 0.296 & .744 & 0.003 \\
Condition*time*group & 3.992 & 1.092 & .362 & 0.021 \\
Emotion*time & 1.029 & 0.256 & .621 & 0.003 \\
Emotion*time*group & 2.057 & 0.606 & .552 & 0.012 \\
Condition*emotion*time & 2 & 0.381 & .684 & 0.004 \\
Condition*emotion*time*group & 3.818 & 1.491 & .209 & 0.029 \\
\hline
\end{tabular}

After applying the additional exclusion criteria for the heart rateanalyses, the sample comprised $n=103$ (PD: $n=44$, AD: $n=28$, HC: $n=31$ )

\section{References}

Achim, A. M., Maziade, M., Raymond, É., Olivier, D., Mérette, C., \& Roy, M. A. (2011). How prevalent are anxiety disorders in schizophrenia? a meta-analysis and critical review on a significant association. Schizophrenia bulletin, 37(4), 811-821. https://doi. org/10.1093/schbul/sbp148.

Ascone, L., Schlier, B., Sundag, J., \& Lincoln, T. M. (2020). Pathways from insecure attachment dimensions to paranoia: the mediating role of hyperactivating emotion regulation versus blaming others. Psychology and Psychotherapy: Theory, Research and Practice, 93(1), 72-87. https://doi.org/10.1111/papt.12208.

Bach, M., Bach, D., de Zwaan, M., \& Serim, M. (1996). Validierung der deutschen Version der 20-item Toronto-Alexithymie-Skala bei Normalpersonen und psychiatrischen Patienten. Psychotherapie Psychosomatik Medizinische Psychologie, 46(1), 23-28.

Bär, K. J., Letzsch, A., Jochum, T., Wagner, G., Greiner, W., \& Sauer, H. (2005). Loss of efferent vagal activity in acute schizophrenia. Journal of Psychiatric Research, 39(5), 519-527. https://doi. org/10.1016/j.jpsychires.2004.12.007.

Barrett, L. F., Gross, J., Christensen, T. C., \& Benvenuto, M. (2001). Knowing what you're feeling and knowing what to do about it: mapping the relation between emotion differentiation and emotion regulation. Cognition \& Emotion, 15(6), 713-724. https://doi. org/10.1080/02699930143000239. 
Benkert, O., \& Hippius, H. (2006). Kompendium der psychiatrischen Pharmakotherapie. Heidelberg: Springer Medizin Verlag.

Benedek, M., \& Kaernbach, C. (2010). A continuous measure of phasic electrodermal activity. Journal of Neuroscience Methods, 190(1), 80-91. https://doi.org/10.1016/j.jneumeth.2010.04.028.

Boucsein, W. (2012). Electrodermal activity. Heidelberg: Springer Science \& Business Media.

Bradley, M. M. (2009). Natural selective attention: orienting and emotion. Psychophysiology, 46(1), 1-11. https://doi.org/10.111 1/j.1469-8986.2008.00702.x.

Der Van Meer, L., Swart, M., Van Der Velde, J., Pijnenborg, G., Wiersma, D., Bruggeman, R., et al. (2014). Neural correlates of emotion regulation in patients with schizophrenia and non-affected siblings. PLoS ONE, 9(6), e99667. https://doi.org/10.1371/journ al.pone.0099667.

Dillon, D. G., \& Pizzagalli, D. A. (2013). Evidence of successful modulation of brain activation and subjective experience during reappraisal of negative emotion in unmedicated depression. Psychiatry Research: Neuroimaging, 212(2), 99-107. https://doi. org/10.1016/j.pscychresns.2013.01.001.

Dudley, R., Taylor, P., Wickham, S., \& Hutton, P. (2015). Psychosis, delusions and the "jumping to conclusions" reasoning bias: a systematic review and meta-analysis. Schizophrenia Bulletin, 42(3), 652-665. https://doi.org/10.1093/schbul/sbv150.

Freeman, D., Emsley, R., Dunn, G., Fowler, D., Bebbington, P., Kuipers, E., et al. (2015). The stress of the street for patients with persecutory delusions: a test of the symptomatic and psychological effects of going outside into a busy urban area. Schizophrenia Bulletin, 41(4), 971-979. https://doi.org/10.1093/schbul/sbu173.

Freeman, D., Garety, P. A., Kuipers, E., Fowler, D., \& Bebbington, P. E. (2002). A cognitive model of persecutory delusions. British Journal of Clinical Psychology, 41(4), 331-347. https://doi. org/10.1348/014466502760387461.

Füstös, J., Gramann, K., Herbert, B. M., \& Pollatos, O. (2012). On the embodiment of emotion regulation: interoceptive awareness facilitates reappraisal. Social Cognitive and Affective Neuroscience, 8(8), 911-917. https://doi.org/10.1093/scan/nss089.

Gardsjord, E. S., Romm, K. L., Friis, S., Barder, H. E., Evensen, J., Haahr, U., et al. (2016). Subjective quality of life in first-episode psychosis. A ten year follow-up study. Schizophrenia Research, 172(1-3), 23-28. https://doi.org/10.1016/j.schres.2016.02.034.

Garety, P. A., Kuipers, E., Fowler, D., Freeman, D., \& Bebbington, P. E. (2001). A cognitive model of the positive symptoms of psychosis. Psychological Medicine, 31(2), 189-195. https://doi. org/10.1017/S0033291701003312.

Garnefski, N., Kraaij, V., \& Spinhoven, P. (2002). Manual for the use of the Cognitive Emotion Regulation Questionnaire. Leiderdorp, The Netherlands: DATEC.

Grezellschak, S., Lincoln, T. M., \& Westermann, S. (2015). Cognitive emotion regulation in patients with schizophrenia: evidence for effective reappraisal and distraction. Psychiatry Research, 229(12), 434-439. https://doi.org/10.1016/j.psychres.2015.05.103.

Gross, J. J. (2015). The extended process model of emotion regulation: elaborations, applications, and future directions. Psychological Inquiry, 26(1), 130-137. https://doi.org/10.1080/10478 40X.2015.989751.

Gross, J. J. (1998). The emerging field of emotion regulation: An integrative review. Review of General Psychology, 2(3), 271. https:// doi.org/10.1037/1089-2680.2.3.271.

Hartley, S., Barrowclough, C., \& Haddock, G. (2013). Anxiety and depression in psychosis: a systematic review of associations with positive psychotic symptoms. Acta Psychiatrica Scandinavica, 128(5), 327-346. https://doi.org/10.1111/acps.12080.

Hofmann, S. G. (2014). Interpersonal emotion regulation model of mood and anxiety disorders. Cognitive Therapy and Research, 38(5), 483-492. https://doi.org/10.1007/s10608-014-9620-1.
Hofmann, S. G., Heering, S., Sawyer, A. T., \& Asnaani, A. (2009). How to handle anxiety: The effects of reappraisal, acceptance, and suppression strategies on anxious arousal. Behaviour Research and Therapy, 47(5), 389-394. https://doi.org/10.1016/j. brat.2009.02.010.

Hofmann, S. G., Sawyer, A. T., Fang, A., \& Asnaani, A. (2012). Emotion dysregulation model of mood and anxiety disorders. Depression and Anxiety, 29(5), 409-416. https://doi.org/10.1002/ da.21888.

Horan, W. P., Hajcak, G., Wynn, J. K., \& Green, M. F. (2013). Impaired emotion regulation in schizophrenia: evidence from event-related potentials. Psychological Medicine, 43(11), 2377-2392. https:// doi.org/10.1017/S0033291713000019.

Huppert, J. D., Weiss, K. A., Lim, R., Pratt, S., \& Smith, T. E. (2001). Quality of life in schizophrenia: contributions of anxiety and depression. Schizophrenia research, 51(2-3), 171-180. https:// doi.org/10.1016/S0920-9964(99)00151-6.

Huq, S. F., Garety, P. A., \& Hemsley, D. R. (1988). Probabilistic judgements in deluded and non-deluded subjects. The Quarterly Journal of Experimental Psychology, 40(4), 801-812. https://doi. org/10.1080/14640748808402300.

Jackson, D. C., Malmstadt, J. R., Larson, C. L., \& Davidson, R. J. (2000). Suppression and enhancement of emotional responses to unpleasant pictures. Psychophysiology, 37(4), 515-522. https:// doi.org/10.1111/1469-8986.3740515.

John, O. P., \& Gross, J. J. (2004). Healthy and unhealthy emotion regulation: personality processes, individual differences, and life span development. Journal of Personality, 72(6), 1301-1334. https:// doi.org/10.1111/j.1467-6494.2004.00298.x.

Kay, S. R., Flszbein, A., \& Opfer, L. A. (1987). The positive and negative syndrome scale (PANSS) for schizophrenia. Schizophrenia Bulletin, 13(2), 261. https://doi.org/10.1093/schbul/13.2.261.

Koole, S. L. (2009). The psychology of emotion regulation: an integrative review. Cognition \& Emotion, 23(1), 4-41. https://doi. org/10.1080/02699930802619031.

Krkovic, K., Clamor, A., Schlier, B., \& Lincoln, T. M. (2019). Emotions and persecutory ideation in daily life: on the trail of the "chicken and egg" problem. Journal of Abnormal Psychology. https://doi.org/10.1037/abn0000495.

Lang, P. J. (2005). International affective picture system (IAPS): affective ratings of pictures and instruction manual. Technical Report, University of Florida

Li, W., Yang, Y., An, F. R., Zhang, L., Ungvari, G. S., Jackson, T., et al. (2020). Prevalence of comorbid depression in schizophrenia: a meta-analysis of observational studies. Journal of Affective Disorders. https://doi.org/10.1016/j.jad.2020.04.056.

Libkuman, T. M., Otani, H., Kern, R., Viger, S. G., \& Novak, N. (2007). Multidimensional normative ratings for the international affective picture system. Behavior Research Methods, 39(2), 326-334. https://doi.org/10.3758/BF03193164.

Lincoln, T. M., Lange, J., Burau, J., Exner, C., \& Moritz, S. (2009). The effect of state anxiety on paranoid ideation and jumping to conclusions. An experimental investigation. Schizophrenia Bulletin, 36(6), 1140-1148. https://doi.org/10.1093/schbul/sbp029.

Lincoln, T. M., Sundag, J., Schlier, B., \& Karow, A. (2018). The relevance of emotion regulation in explaining why social exclusion triggers paranoia in individuals at clinical high risk of psychosis. Schizophrenia Bulletin, 44(4), 757-767. https://doi.org/10.1093/ schbul/sbx 135 .

Lincoln, T. M., Köther, U., Hartmann, M., Kempkensteffen, J., \& Moritz, S. (2015). Responses to stress in patients with psychotic disorders compared to persons with varying levels of vulnerability to psychosis, persons with depression and healthy controls. Journal of Behavior Therapy and Experimental Psychiatry, 47, 92-101. https://doi.org/10.1016/j.jbtep.2014.11.011. 
Lincoln, T. M., Ziegler, M., Mehl, S., Kesting, M.-L., Lüllmann, E., Westermann, S., et al. (2012). Moving from efficacy to effectiveness in cognitive behavioral therapy for psychosis: a randomized clinical practice trial. Journal of Consulting and Clinical Psychology, 80(4), 674. https://doi.org/10.1037/a0028665.

Loch, N., Hiller, W., \& Witthöft, M. (2011). Der Cognitive Emotion Regulation Questionnaire (CERQ). Zeitschrift Für Klinische Psychologie Und Psychotherapie, 40, 94-106. https://doi. org/10.1026/1616-3443/a000079.

Ludwig, L., Mehl, S., Krkovic, K., \& Lincoln, T. M. (2020). Effectiveness of emotion regulation in daily life in individuals with psychosis and non-clinical controls - an experience sampling study. Journal of Abnormal Psychology, 129(4), 408-421. https://doi. org/10.1037/abn0000505.

Ludwig, L., Werner, D., \& Lincoln, T. M. (2019). The relevance of cognitive emotion regulation to psychotic symptoms-A systematic review and meta-analysis. Clinical Psychology Review. https://doi. org/10.1016/j.cpr.2019.101746.

McRae, K. (2013). Emotion regulation frequency and success: separating constructs from methods and time scale. Social and Personality Psychology Compass, 7(5), 289-302. https://doi.org/10.1111/ spc3.12027.

McRae, K., Jacobs, S. E., Ray, R. D., John, O. P., \& Gross, J. J. (2012a). Individual differences in reappraisal ability: Links to reappraisal frequency, well-being, and cognitive control. Journal of Research in Personality, 46(1), 2-7. https://doi.org/10.1016/j. jrp.2011.10.003.

McRae, K., Ciesielski, B., \& Gross, J. J. (2012b). Unpacking cognitive reappraisal: goals, tactics, and outcomes. Emotion, 12(2), 250. https://doi.org/10.1037/a0026351.

Mehl, S., Schlier, B., \& Lincoln, T. M. (2018). Does CBT for psychosis have an impact on delusions by improving reasoning biases and negative self-schemas? Zeitschrift Für Psychologie, 226, 152163. https://doi.org/10.1027/2151-2604/a000335.

Modinos, G., Ormel, J., \& Aleman, A. (2010). Altered activation and functional connectivity of neural systems supporting cognitive control of emotion in psychosis proneness. Schizophrenia Research, 118(1-3), 88-97. https://doi.org/10.1016/j.schre s.2010.01.030.

Moritz, S., Veckenstedt, R., Hottenrott, B., Woodward, T. S., Randjbar, S., \& Lincoln, T. M. (2010). Different sides of the same coin? intercorrelations of cognitive biases in schizophrenia. Cognitive Neuropsychiatry, 15(4), 406-421. https://doi.org/10.1080/13546 800903399993.

Oliver, J. E., O'Connor, J. A., Jose, P. E., McLachlan, K., \& Peters, E. (2012). The impact of negative schemas, mood and psychological flexibility on delusional ideation-mediating and moderating effects. Psychosis, 4(1), 6-18. https://doi.org/10.1080/17522 439.2011.637117.

O'Driscoll, C., Laing, J., \& Mason, O. (2014). Cognitive emotion regulation strategies, alexithymia and dissociation in schizophrenia, a review and meta-analysis. Clinical Psychology Review, 34(6), 482-495. https://doi.org/10.1016/j.cpr.2014.07.002.

Painter, J. M., Stellar, J. E., Moran, E. K., \& Kring, A. M. (2019). A multicomponent approach toward understanding emotion regulation in schizophrenia. Journal of Clinical Psychology, 75(1), 178-189. https://doi.org/10.1002/jclp.22698.

Perry, Y., Henry, J. D., Nangle, M. R., \& Grisham, J. R. (2012). Regulation of negative affect in schizophrenia: the effectiveness of acceptance versus reappraisal and suppression. Journal of Clinical and Experimental Neuropsychology, 34(5), 497-508. https:// doi.org/10.1080/13803395.2012.661405.

Phillips, L. K., \& Seidman, L. J. (2008). Emotion processing in persons at risk for schizophrenia. Schizophrenia bulletin, 34(5), 888-903. https://doi.org/10.1093/schbul/sbn085.
Picó-Pérez, M., Radua, J., Steward, T., Menchón, J. M., \& SorianoMas, C. (2017). Emotion regulation in mood and anxiety disorders: a meta-analysis of fMRI cognitive reappraisal studies. Progress in Neuro-Psychopharmacology and Biological Psychiatry, 79, 96-104. https://doi.org/10.1016/j.pnpbp.2017.06.001.

Pruessner, L., Barnow, S., Holt, D. V., Joormann, J., \& Schulze, K. (2020). A cognitive control framework for understanding emotion regulation flexibility. Emotion. https://doi.org/10.1037/emo00 00658.

Ray, R. D., McRae, K., Ochsner, K. N., \& Gross, J. J. (2010). Cognitive reappraisal of negative affect: converging evidence from EMG and self-report. Emotion, 10(4), 587. https://doi.org/10.1037/ a0019015.

Rechlin, T., Claus, D., \& Weis, M. (1994). Heart rate variability in schizophrenic patients and changes of autonomic heart rate parameters during treatment with clozapine. Biological Psychiatry, 35(11), 888-892.

Reitan, R. M. (1958). Validity of the Trail Making Test as an indicator of organic brain damage. Perceptual and Motor Skills, 8(3), 271-276.

Schmeichel, B. J., \& Tang, D. (2015). Individual differences in executive functioning and their relationship to emotional processes and responses. Current Directions in Psychological Science, 24(2), 93-98. https://doi.org/10.1177/0963721414555178.

Sheehan, D. V., Lecrubier, Y., Sheehan, K. H., Amorim, P., Janavs, J., Weiller, E., et al. (1998). The Mini-International Neuropsychiatric Interview (MINI): the development and validation of a structured diagnostic psychiatric interview for DSM-IV and ICD-10. The Journal of Clinical Psychiatry, 59(Suppl 20), 22-33.

Söder, E., Clamor, A., Kempkensteffen, J., Moritz, S., \& Lincoln, T. M. (2018). Stress levels in psychosis: do body and mind diverge? Biological Psychology, 138, 156-164. https://doi.org/10.1016/j. biopsycho.2018.08.016.

Stemmler, G., Heldmann, M., Pauls, C. A., \& Scherer, T. (2001). Constraints for emotion specificity in fear and anger: the context counts. Psychophysiology, 38(2), 275-291. https://doi. org/10.1111/1469-8986.3820275.

Strauss, G. P., Kappenman, E. S., Culbreth, A. J., Catalano, L. T., Lee, B. G., \& Gold, J. M. (2013). Emotion regulation abnormalities in schizophrenia: cognitive change strategies fail to decrease the neural response to unpleasant stimuli. Schizophrenia Bulletin, 39(4), 872-883. https://doi.org/10.1093/schbul/sbs186.

Sundag, J., Ascone, L., \& Lincoln, T. M. (2018). The predictive value of early maladaptive schemas in paranoid responses to social stress. Clinical Psychology \& Psychotherapy, 25(1), 65-75. https ://doi.org/10.1002/cpp.2128.

Tamir, M., Bigman, Y. E., Rhodes, E., Salerno, J., \& Schreier, J. (2015). An expectancy-value model of emotion regulation: implications for motivation, emotional experience, and decision making. Emotion, 15(1), 90. https://doi.org/10.1037/emo0000021.

Thewissen, V., Bentall, R. P., Oorschot, M., van Lierop, T., van Os, J., \& Myin-Germeys, I. (2011). Emotions, self esteem, and paranoid episodes: an experience sampling study. British Journal of Clinical Psychology, 50(2), 178-195. https://doi.org/10.1348/01446 $6510 X 508677$.

Thompson, R. A. (1994). Emotion regulation: a theme in search of definition. Monographs of the Society for Research in Child Development, 59(2-3), 25-52. https://doi.org/10.1111/j.1540-5834.1994. tb01276.x.

Valkonen-Korhonen, M., Tarvainen, M. P., Ranta-Aho, P., Karjalainen, P. A., Partanen, J., Karhu, J., et al. (2003). Heart rate variability in acute psychosis. Psychophysiology, 40(5), 716-726. https://doi. org/10.1111/1469-8986.00072.

Van Rijn, S., Schothorst, P., van't Wout, M., Sprong, M., Ziermans, T., van Engeland, H., et al. (2011). Affective dysfunctions in adolescents at risk for psychosis: emotion awareness and social 
functioning. Psychiatry Research, 187(1), 100-105. https://doi. org/10.1016/j.psychres.2010.10.007.

Visser, K. F., Esfahlani, F. Z., Sayama, H., \& Strauss, G. P. (2018). An ecological momentary assessment evaluation of emotion regulation abnormalities in schizophrenia. Psychological Medicine, 48(14), 2337-2345. https://doi.org/10.1017/S0033291717003865.

Westermann, S., Rief, W., \& Lincoln, T. M. (2014). Emotion regulation in delusion-proneness: deficits in cognitive reappraisal, but not in expressive suppression. Psychology and Psychotherapy: Theory, Research and Practice, 87(1), 1-14. https://doi.org/10.1111/ papt. 12000.
Wolgast, M., Lundh, L.-G., \& Viborg, G. (2011). Cognitive reappraisal and acceptance: an experimental comparison of two emotion regulation strategies. Behaviour Research and Therapy, 49(12), 858-866. https://doi.org/10.1016/j.brat.2011.09.011.

Publisher's Note Springer Nature remains neutral with regard to jurisdictional claims in published maps and institutional affiliations. 